\begin{tabular}{|c|l|}
\hline Title & Coil-globule transition of poly (methyl methacrylate) in a mixed solvent \\
\hline Author(s) & Nakata, Mitsuo \\
\hline Citation & $\begin{array}{l}\text { PHY SICAL REVIEW E, 51(6), 5770-5775 } \\
\text { https://doi.org/10.1103/PhySRevE.51.5770 }\end{array}$ \\
\hline Issue Date & 1995-06 \\
\hline Doc URL & http://hdl.handle.net/2115/5860 \\
\hline Rights & Copyright $\odot$ 1995 A merican Physical Society \\
\hline Type & article \\
\hline File Information & PRE51-6.pdf \\
\hline
\end{tabular}

Instructions for use 


\title{
Coil-globule transition of poly(methyl methacrylate) in a mixed solvent
}

\author{
Mitsuo Nakata \\ Department of Polymer Science, Faculty of Science, Hokkaido University, Sapporo 060, Japan
}

(Received 25 January 1995)

\begin{abstract}
From light scattering measurements the coil-globule transition has been observed for poly(methyl methacrylate) in the mixed solvent water +tert-butyl alcohol with molecular weight $\boldsymbol{M}_{w}=2.38 \times 10^{6}$. Since the phase separation occurred very slowly for this system, the mean square radius of gyration $\left\langle s^{2}\right\rangle$ and the second virial coefficient $A_{2}$ were determined reliably even far below the phase-separation temperature. A comparison of the observed $\left\langle s^{2}\right\rangle$ with a theory for a contracted coil confirmed the coilglobule transition. The observed second virial coefficient had a minimum in the temperature region where $\left\langle s^{2}\right\rangle$ approaches the value for the globule state.
\end{abstract}

PACS number(s): $36.20 . \mathrm{Ey}, 61.25 . \mathrm{Hq}$

\section{INTRODUCTION}

To investigate the collapse of a polymer coil below the $\theta$ temperature, Ptitsyn, Kron, and Eizner first introduced a term caused by the third virial coefficient in the Flory equation [1] for the expansion factor $\alpha$ as [2]

$$
\alpha^{5}-\alpha^{3}-y / \alpha^{3}=x
$$

where $y$ stems from the third virial coefficient and is considered to be a constant independent of the temperature $T$ and the molecular weight $M$, and $x$ is equal to the excluded volume parameter $z \propto \tau M^{1 / 2}$ except for a numerical constant. Here, $\tau$ is given by $\tau=1-\theta / T$. For $T \ll \theta$ Eq. (1) gives the asymptotic relation $-x \alpha^{3} \sim y$, which means that the monomer density inside the polymer domain is constant independent of $M$. Thus Eq. (1) predicts a coil-globule transition similar to the condensation of a real gas. "This intriguing behavior of Eq. (1) stimulated many theoretical [3-8] and experimental studies [9-17]. However, in experiments on dilute solutions of synthetic polymers the coil-globule transition is obscured by the phase separation. In the usual experimental studies a cloud point due to phase separation appears before collapse of the polymer coils because of the same nature of the intra- and intermolecular interactions. Therefore experiments to detect the coil-globule transition were made for peculiar polymers $[10,14]$ or at extreme conditions [11-13]. Otherwise, experimental data were obtained examining the effect of phase separation [9,15-17].

The coil-globule transition was first observed for polyacrylamide molecules in acetone + water mixtures by varying the composition [10]; a covalently cross-linked polyacrylamide network had been known to collapse reversibly in the mixtures. A straightforward way to observe a coil collapse for common polymers is to carry out experiments at extremely low concentrations. Tanaka and co-workers observed a collapse of polystyrene (PS) coils with the molecular weight $M_{w}=2.7 \times 10^{7}$ in cyclohexane at concentrations as low as $10^{-7} \mathrm{~g} / \mathrm{ml}[11,12]$. Measurements on PS solutions have also been made at higher concentrations such as $\sim 10^{-6}$ or $10^{-5} \mathrm{~g} / \mathrm{ml}$. Stepanek, Konak, and Sedlacek employed the viscous solvent dioc- tyl phthalate to suppress the phase separation and observed a collapse for PS coils in the metastable region [13]. Chu and co-workers made light scattering measurements in the range from the $\theta$ temperature to near the phase-separation temperature [15-17]. According to the data analysis with a scaling law, the transition to a globule state was suggested to occur at relatively large $\alpha$, and the data observed in a metastable region deviated largely from the master curve expected from the scaling law [17]. Grosberg and Kuznetsov [18] scrutinized the experimental data mentioned above based on their theory [8] and obtained the following conclusions. The scaling law $\alpha \sim x^{-1 / 3}$ is not considered as an adequate criterion for the globule state in the analysis of experimental data. The observation of the globule state made by Swislow and co-workers $[11,12]$ is inferred to be made below the phase-separation temperature. The globule state depends on the value of $y$ and cannot be decided by a general criterion. Thus, for the present, the coil-globule transition cannot be concluded to be a universal phenomenon for polymer coils from the above sparse experimental studies which were made at extreme conditions and gave different descriptions of the globule state.

Birshtein and Pryamitsyn indicated that the Flory equation for $\alpha$ cannot be extended to the globule state because of the different segment distributions about the center of mass in expanded and contracted coils [7]. For the contracted coil they proposed a novel expression for the entropy force and derived an equation for $\alpha$ as

$$
\alpha^{3}-\alpha-C\left(\alpha^{-3}-1\right)=B M^{1 / 2} \tau,
$$

where $B$ and $C$ are constants independent of $M$ and $T$ and $C$ is caused by the third virial coefficients. The asymptotic relation of Eq. (2) for $\alpha<1$ also predicts the globule state. Equation (2) was shown to agree with the experimental data below the $\theta$ temperature $[12,13]$. Both Eqs. (1) and (2) have a critical point below which the coilglobule transition becomes discontinuous. For Eq. (1) the critical point appears at $y=0.02278$ and $x=-0.2415$, while for Eq. (2) it appears at $C=0.005487$ and $B M^{1 / 2} \tau=-0.4135$. The value of $\alpha$ at the critical point is different for Eqs. (1) and (2) as 0.6708 and 0.4714 , respec- 
tively.

In this work, the coil-globule transition was studied for poly(methyl methacrylate) (PMMA) in the mixed solvent water+tert-butyl alcohol $(\mathrm{BuOH})$ by light scattering measurements. Since phase separation occurred very slowly for this system, reliable determinations were made for the second virial coefficient $A_{2}$ and the mean square radius of gyration $\left\langle s^{2}\right\rangle$ even far below the phaseseparation temperature. The expansion factor observed below the $\theta$ temperature coincided with Eq. (2) and indicated the coil-globule transition. The observed second virial coefficient had a minimum near the crossover point between the coil and globule regions, and was compared with a theoretical calculation [19] and an experimental study [9].

\section{EXPERIMENT AND RESULTS}

PMMA samples were prepared by bulk polymerization of the monomer and purified by solution fractionation in the same conditions as in a previous work [20]. For the present study we used a middle fraction with the weightaveraged molecular weight $M_{w}=2.38 \times 10^{6}$ and the molecular weight distribution $M_{w} / M_{n} \simeq 1.20$. The $\mathrm{BuOH}$ was fractionally distilled immediately before use. The water was purified by a standard method.

PMMA is completely immiscible with water and partially soluble in $\mathrm{BuOH}$ at high temperature. However, PMMA becomes miscible with the mixed solvent water $+\mathrm{BuOH}$ in the composition range $0.65<u_{2}<0.99$, where $u_{2}$ is the volume fraction of $\mathrm{BuOH}$ in the mixed solvent [21]. The present study on the coil-globule transition was performed with the marginal solvent at $u_{2}=0.975$ by varying the temperature.

The refractive index of the mixture water $+\mathrm{BuOH}$ was measured as a function of $u_{2}$ and the derivative $d n / d u_{2}$ was found to vanish near $u_{2}=0.975$. The refractive index increment $(\partial n / \partial c)_{u_{2}}$ at $u_{2}=0.975$ was determined by a differential refractometer as $0.0994+3.2 \times 10^{-4} t$, where $c$ is the polymer concentration in the solution and $t$ is the temperature in ${ }^{\circ} \mathrm{C} . d n / d u_{2}$ was negligibly small compared with $(\partial n / \partial c)_{u_{2}}$. Thus the mixed solvent behaved as a single solvent in light scattering and the excess Rayleigh's ratio $R_{\theta}$ from the polymer can be given by the usual scattering equation [22]

$\left(K c / R_{\theta}\right)^{1 / 2}=M_{w}^{-1 / 2}+A_{2} M_{w}^{1 / 2} c+\frac{1}{6}\left\langle s^{2}\right\rangle M_{w}^{-1 / 2} q^{2}$

with $K=\left(2 \pi^{2} n^{2} / N_{A} \lambda^{4}\right)(\partial n / \partial c)_{u_{2}}^{2} \quad$ and $\quad q=(4 \pi n /$ $\lambda) \sin (\theta / 2)$, where $N_{A}$ is Avogadro's number and $\lambda$ is the wavelength of incident light in vacuum. $A_{2}$ and $\left\langle s^{2}\right\rangle$ were determined by a Zimm plot according to Eq. (3).

Light scattering measurements were carried out at angular intervals of $15^{\circ}$ in the range from $30^{\circ}$ to $135^{\circ}$ with unpolarized incident light $(\lambda=435.8 \mathrm{~nm})$ [22]. Four solutions of different PMMA concentrations $(\mathrm{g} / \mathrm{ml})$ near $c \times 10^{4}=1.2,2.4,3.6$, and 4.8 were used for the measurements. For optical clarification each solution was filtered twice through a Sartorius membrane filter (SM116, 0.45 or $0.8 \mu \mathrm{m}$ ) into an optical cell of $18 \mathrm{~mm}$ i.d. with $1 \mathrm{~mm}$ glass thickness and kept in the dark at a few kelvins above the phase-separation temperature $T_{p}$. For all the solutions $T_{p}$ was observed between 36.0 and $37.0^{\circ} \mathrm{C}$. The optical cell was immersed in a cylindrical cell kept at a constant temperature within $\pm 0.01 \mathrm{~K}$ and the scattered intensities were measured at $30 \mathrm{~min}$ after the setup of the cell for the sake of thermal equilibrium.

In Fig. 1 the light scattering data at 41.6 and $28.0^{\circ} \mathrm{C}$ are given by plotting $\left(K c / R_{\theta}\right)^{1 / 2}$ against $\sin ^{2}(\theta / 2)$ $+1000 c$ in the form of a Zimm plot. At each temperature the plot of $\left(K c / R_{\theta}\right)^{1 / 2}$ vs $\sin ^{2}(\theta / 2)$ is described by parallel straight lines. The filled circles represent the extrapolation to zero angle and are used for the extrapolation to zero concentration. The intercepts at $c=0$ and $\theta=0$ of the plots at the two temperatures agree with each other within an error of $4 \%$, indicating the correct value of $M_{w}$. At $28.0^{\circ} \mathrm{C}$ further measurements were carried out for the solution of $c=4.8 \times 10^{-4}$ at 60 and 90 min after the setup of the cell. In Fig. 1 the curves $a$ and $b$ represent the data at 60 and $90 \mathrm{~min}$, respectively. The gradual increase of scattered intensities with time exhibits the onset of phase separation. The straight lines for the plots $a$ and $b$ are illustrated to run parallel with those of the plots at $30 \mathrm{~min}$.

In Fig. 2 the light scattering data at $34.0^{\circ} \mathrm{C}$ are given in the same way as those at $28.0^{\circ} \mathrm{C}$ in Fig. 1. The data at 30 min are extrapolated to $\theta=0$ and to $c=0$, and the inter-

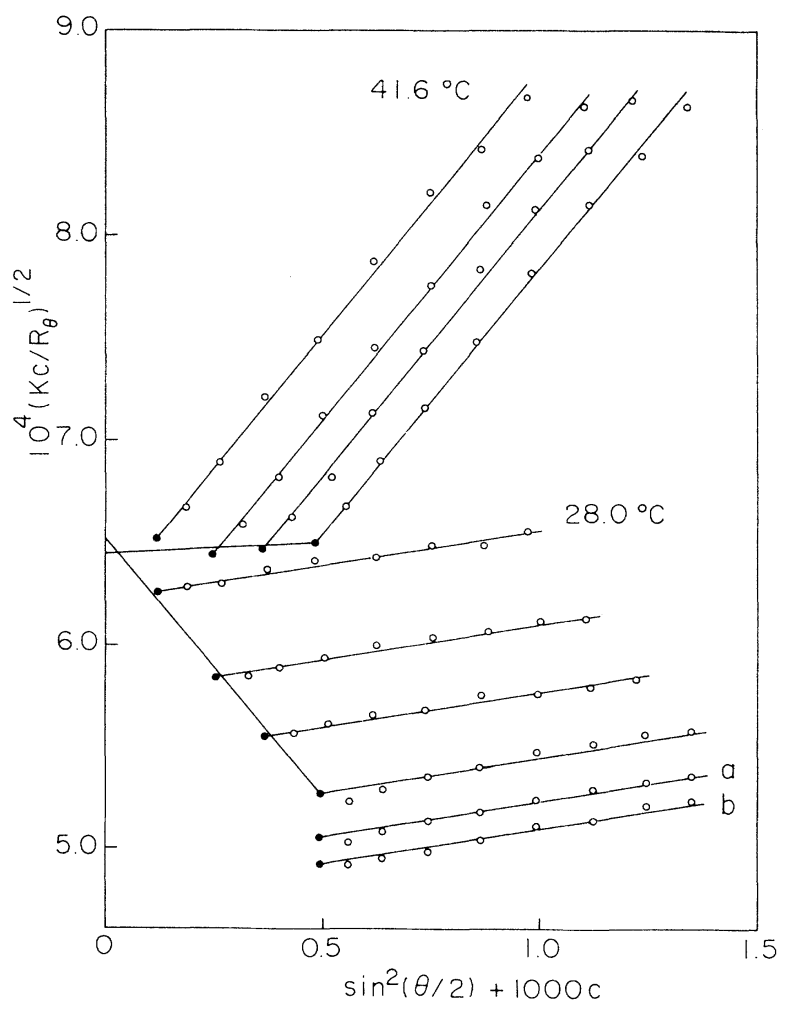

FIG. 1. Zimm plots for the light scattering data obtained at the indicated temperatures at $30 \mathrm{~min}$ after setup of the solution. The filled circles represent extrapolation to zero angle. The plots $a$ and $b$ were obtained at 60 and 90 min after setup of the solution, respectively. 


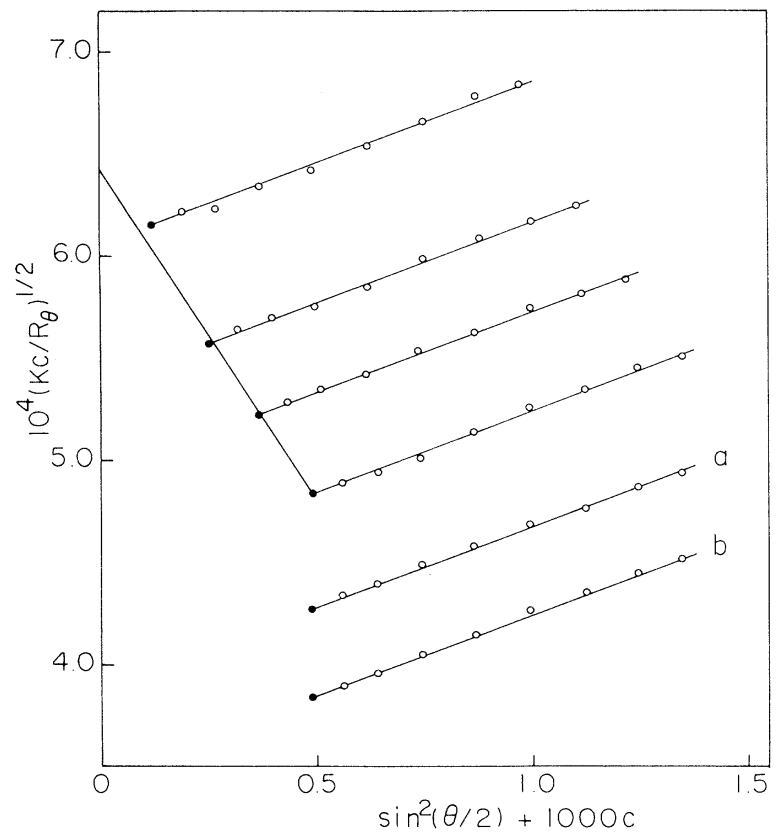

FIG. 2. Zimm plot for the light scattering data obtained at $34.0^{\circ} \mathrm{C}$ at $30 \mathrm{~min}$ after setup of the solution. Symbols are the same as in Fig. 1.

cept gives the molecular weight correctly as $M_{w}=2.32 \times 10^{6}$. The plots $a$ and $b$ obtained for the data at 60 and $90 \mathrm{~min}$, respectively, indicate a considerable increase of scattered intensity with time. However, all the plots of $\left(K c / R_{\theta}\right)^{1 / 2}$ vs $\sin ^{2}(\theta / 2)$ are described by a straight line with the same slope. Thus the mean square radius of gyration $\left\langle s^{2}\right\rangle$ can be evaluated reasonably from the slope of $\left(K c / R_{\theta}\right)^{1 / 2}$ vs $\sin ^{2}(\theta / 2)$ even below the phase-separation temperature. The correct intercept at $c=0$ and $\theta=0$ means that the effect of the phase separation vanishes on extrapolating to zero concentration. The straight lines of the plot of $\left(K c / R_{\theta}\right)^{1 / 2}$ vs $c$ at $\theta=0$ suggest that the rate of phase separation decreases linearly with decreasing concentration. The rate was most noticeable near $34.0^{\circ} \mathrm{C}$. The solution which had been kept below $T_{p}$ for a time long enough for measurements recovered its normal scattering intensity without delay,

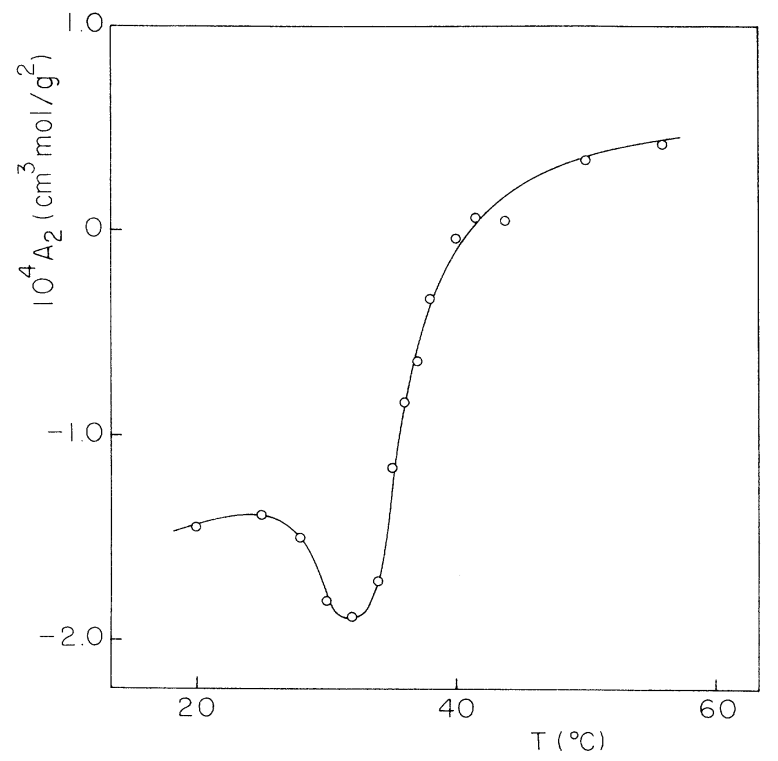

FIG. 3. Second virial coefficient $A_{2}$ vs temperature.

when the temperature was raised up above $T_{p}$.

The data at 60 and at 90 min suggest that the slope of the plot $\left(K c / R_{\theta}\right)^{1 / 2}$ vs $c$ at $\theta=0$ is affected by the phase separation. However, the straight line and the correct intercept of the plot allow us to determine the second virial coefficient $A_{2}$ by estimating the slope at zero time with the data at 60 and $90 \mathrm{~min}$.

In Table I the obtained data of $\left\langle s^{2}\right\rangle$ and $A_{2}$ are summarized. Figure 3 shows the plot of $A_{2}$ vs temperature $\left({ }^{\circ} \mathrm{C}\right)$. The open circles indicate data points and the solid line is drawn in accord with the data points by eye. $A_{2}$ vanishes at $41.5^{\circ} \mathrm{C}$ and has a minimum near $32.0^{\circ} \mathrm{C}$. Figure 4 shows the plot of $\left\langle s^{2}\right\rangle$ vs temperature $\left({ }^{\circ} \mathrm{C}\right)$ with the open circles as the data points. $\left\langle s^{2}\right\rangle$ decreases rapidly with decreasing temperature with a slow down below $30^{\circ} \mathrm{C}$. The plot for $\left\langle s^{2}\right\rangle$ does not have a depression as observed in the plot for $A_{2}$. At $41.5^{\circ} \mathrm{C}\left\langle s^{2}\right\rangle$ was evaluated to be $1.46 \times 10^{-11} \mathrm{~cm}^{2}$, which is close to the value $1.51 \times 10^{-11} \mathrm{~cm}^{2}$ determined at the $\theta$ temperature with the solvent 1-chlorobutane. This agreement means that the mixed solvent water $+\mathrm{BuOH}$ acts like a single solvent

TABLE I. Mean square radius of gyration $\left\langle s^{2}\right\rangle$ and second virial coefficient $A_{2}$ observed for poly(methyl methacrylate) in the mixed solvent water + tert-butyl alcohol.

\begin{tabular}{cccccc}
\hline $\begin{array}{c}\text { Temp. } \\
\left({ }^{\circ} \mathrm{C}\right)\end{array}$ & $\begin{array}{c}10^{11}\left\langle\mathrm{~s}^{2}\right\rangle \\
\left(\mathrm{cm}^{2}\right)\end{array}$ & $\begin{array}{c}10^{5} A_{2} \\
\left(\mathrm{~cm}^{3} \mathrm{~mol} / \mathrm{g}^{2}\right)\end{array}$ & $\begin{array}{c}\text { Temp. } \\
\left({ }^{\circ} \mathrm{C}\right)\end{array}$ & $\begin{array}{c}10^{11}\left\langle\mathrm{~s}^{2}\right\rangle \\
\left(\mathrm{cm}^{2}\right)\end{array}$ & $\begin{array}{c}10^{5} A_{2} \\
\left(\mathrm{~cm}^{3} \mathrm{~mol}^{2} / \mathrm{g}^{2}\right)\end{array}$ \\
\hline 55.8 & 2.35 & 4.1 & 35.0 & 0.57 & -11.6 \\
50.0 & 2.06 & 3.5 & 34.0 & 0.46 & -17.1 \\
43.8 & 1.64 & 0.5 & 32.0 & 0.30 & -18.9 \\
41.6 & 1.52 & 0.6 & 30.0 & 0.25 & -18.1 \\
40.0 & 1.27 & -0.4 & 28.0 & 0.20 & -13.9 \\
38.0 & 1.05 & -3.4 & 25.0 & 0.17 & -14.5 \\
37.0 & 0.91 & -6.4 & 20.0 & 0.16 & \\
36.0 & 0.70 & -8.4 & & & \\
\hline \hline
\end{tabular}




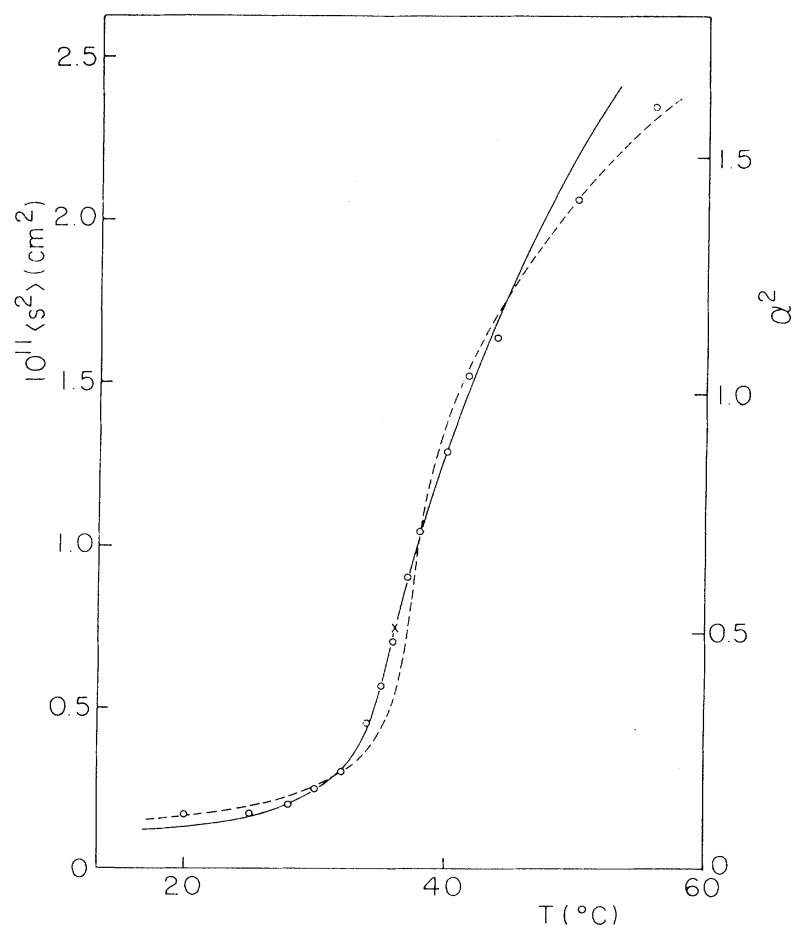

FIG. 4. Mean square radius of gyration $\left\langle s^{2}\right\rangle$ or square of expansion factor $\alpha^{2}$ against temperature. The open circles are for experimental data. The solid and broken lines are described by Eqs. (2) and (1), respectively. The cross indicates a coil-globule transition point.

for PMMA without preferential sorption [22] and the temperature $41.5^{\circ} \mathrm{C}$ can be taken as the $\theta$ temperature for the mixed solvent. Thus the expansion factor $\alpha^{2}=\left\langle s^{2}\right\rangle /\left\langle s^{2}\right\rangle_{0}$ was calculated with the unperturbed dimension $\left\langle s^{2}\right\rangle_{0}=1.46 \times 10^{-11}$. In Fig. 4 the axis at the right gives $\alpha^{2}$.

\section{COMPARISON WITH THEORIES}

The observed behavior of $\alpha$ was compared with Eqs. (1) and (2). According to Eq. (2) the plot of $\left(\alpha^{3}-\alpha\right) /\left(1-\alpha^{-3}\right)$ vs $\tau M^{1 / 2} /\left(1-\alpha^{-3}\right)$ for $\alpha<1$ should be linear and the slope and the intercept yield $B$ and $C$, respectively. Though Birshtein and Pryamitsyn defined $\tau$ as $(T-\theta) / \theta$, we use the definition $\tau=(T-\theta) / T$ because of a minor difference in this study. Figure 5 shows the plot for the present data for $\alpha<1$. As expected from Eq. (2) the data points fall on a straight line, which gives $B=0.0160$ and $C=0.044$. In Fig. 4 the solid curve is described by Eq. (2) with these values of $B$ and $C$. In Fig. $6,-\alpha^{3} \tau M_{w}^{1 / 2}$ is plotted against $-\tau M_{w}^{1 / 2}$ to observe the transition to the globule state corresponding to $-\alpha^{3} \tau M_{w}^{1 / 2}=C / B=2.75$. The solid line gives the calculation due to Eq. (2) and the open circles represent the experimental data. The horizontal line at the right indicates the asymptotic value $C / B=2.75$. The solid curve approaches the asymptotic limit closely for $-\tau M_{w}^{1 / 2}>70$. Birshtein and Pryamitsyn assigned the crossover point

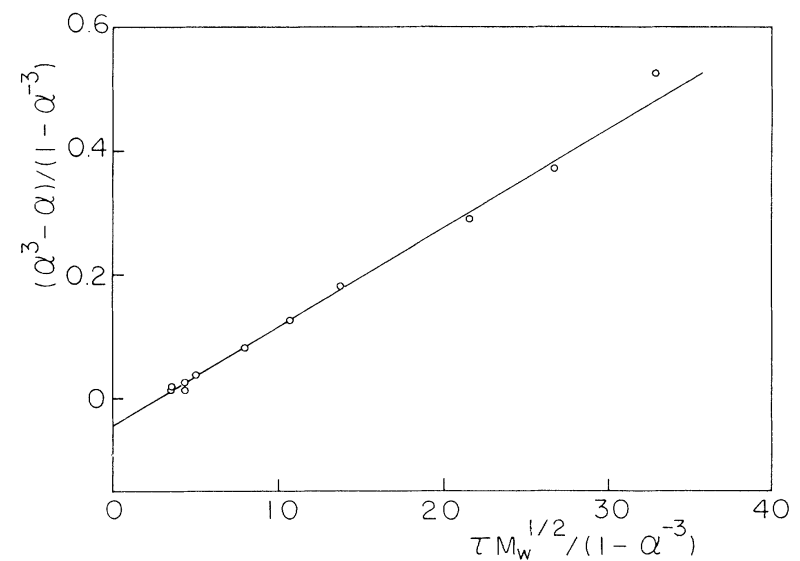

FIG. 5. Plot due to Eq. (2). The intercept and slope of the straight line give $C=0.044$ and $B=0.0160$ for the constants in Eq. (2), respectively.

between the coil and globule regions by the balance of the two terms in Eq. (2), i.e., $\alpha-a^{3}=C\left(\alpha^{-3}-1\right)$. For the present case of $C=0.044$ this balance is obtained at $\alpha^{2}=0.2256$ or $\tau M_{w}^{1 / 2}=-45.9$. In light of the plots in Figs. 5 and 6 this crossover point seems to put too much weight on the globule state. For the critical value $C=0.005487$ the crossover point should coincide with the critical point at $\alpha=0.4714$. These values of $C$ and $\alpha$ yield $C\left(\alpha^{-3}-1\right) /\left(\alpha-\alpha^{3}\right)=0.128$, which implies that the coil-globule transition is caused by a small contribution from the third virial coefficient. Therefore it is practical

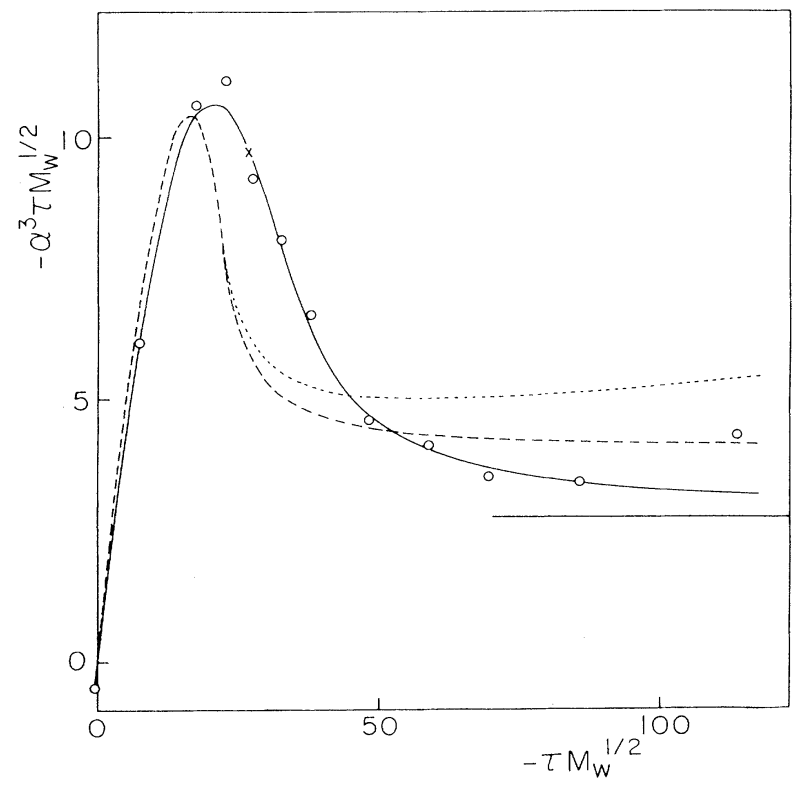

FIG. 6. Plot of $-\alpha^{3} \tau M_{w}^{1 / 2}$ vs $-\tau M_{w}^{1 / 2}$ below the $\theta$ temperature. The open circles are for experimental data. The solid and broken lines are for Eqs. (2) and (1), respectively. The dotted line represents deviation of the Sanchez theory from Eq. (1). The horizontal line at the right indicates the asymptotic limit $C / B=2.75$ of Eq. (2). The cross is a crossover point. 
to specify the crossover point by $d^{2} \alpha^{k} / d p^{2}=\infty$, which is one of the critical conditions. Here, $k$ is an integer and $p=B \tau M_{w}^{1 / 2}$. For $C=0.044$ the crossover point depends somewhat on the $k$ value. We estimated the crossover point tentatively for $k=2$ as $\alpha^{2}=0.511$ or $\tau M^{1 / 2}=-26.7$, which is indicated by the cross in Figs. 4 and 6.

According to Eq. (1) the plot of $\alpha^{8}-\alpha^{6}$ vs $\alpha^{3} \tau$ should give a straight line. However, the present data did not yield a straight line for the plot. In Figs. 4 and 6 the broken line depicts Eq. (1) with $x=26.0 \tau$ and $y=0.068$, which were determined by trial and error. For $\alpha<1$ the agreement between the data and Eq. (1) is not quantitative.

The segment volume fraction $\phi$ in the polymer domain may be given by

$$
\phi=\left(M_{w} / \rho\right) /\left\{(4 \pi / 3)\left\langle s^{2}\right\rangle^{3 / 2} N_{A}\right\},
$$

where $\rho$ is the density of PMMA in the liquid state. At $25^{\circ} \mathrm{C} \phi$ is evaluated as 0.36 with the experimental data $\left\langle s^{2}\right\rangle=0.17 \times 10^{-11} \mathrm{~cm}^{2}$ and $\rho=1.20 \mathrm{~g} / \mathrm{ml}$. The use of $\left\{\frac{5}{3}\left\langle s^{2}\right\rangle\right\}^{1 / 2}$ for the radius of the globule reduces $\phi$ to $\phi=0.17$. At low temperatures the segment fraction is not dilute but concentrated in the polymer domain.

For a polymer coil of high segment fraction Sanchez theory may be better than Eq. (1). The modified Sanchez theory [12] can be expressed in the form of Eq. (1) with $x=\left(3 r \alpha_{m}^{3} / 14\right) \sigma \tau$ and $y=\left(r \alpha_{m}^{6} / 7\right) Y\left(\alpha_{m}^{3} / \alpha^{3}\right)$, where $r$ is the number of statistical segments, $\sigma$ is a thermodynamic factor, $\alpha_{m}$ is the expansion factor for the totally collapsed state, and $Y\left(\alpha_{m}^{3} / \alpha^{3}\right)$ has the functional form

$$
Y(\phi)=-3\left\{\ln (1-\phi)+\phi+\frac{1}{2} \phi^{2}\right\} / \phi^{3} .
$$

Since $Y(\phi)$ becomes unity for $\phi=0$, the Sanchez theory tends to agree with Eq. (1) for $\alpha>1$. With the plausible assumption $\alpha_{m}^{3}=\phi \alpha^{3}, \alpha_{m}^{3}$ can be estimated to be 0.0141 . Then we obtain $r=10^{-3} M_{w}$ from the expression for $y$ with $Y(\phi)=1$, and $\sigma=3.6$ from the expression for $x$. The Sanchez theory deviates from Eq. (1) for small $\alpha$ as shown by the dotted line in Fig. 6. The upward trend of the dotted line at large $-\tau M_{w}^{1 / 2}$ seems to be compatible with the trend of the data, though the Sanchez theory does not improve Eq. (1) essentially. The present value of $\sigma$ and the relation between $r$ and $M_{w}$ are compared with $\sigma=4$ and $r=9.6 \times 10^{-4} M_{w}$ for polystyrene in cyclohexane [12]. The data point at the lowest temperature deviates from the solid line in Figs. 4 and 6. It is not certain whether this deviation is caused by an experimental error or the effect of high segment fraction in the polymer domain.

A plot similar to that in Fig. 6 has been made for various experimental data of polystyrene in Fig. 11 in Ref. [15]. In this plot a peak is observed for the data in cyclohexane $[11,12]$ and in dioctyl phthalate [13]. Except for these data the plot appears to be made for data obtained in the coil state.

In Fig. 3 the plot of $A_{2}$ vs temperature has a marked depression at $32^{\circ} \mathrm{C}$. On the lower temperature side of the depression $A_{2}$ does not seem to increase with decreasing temperature. Tanaka [19] made a theoretical study of $A_{2}$ below the $\theta$ temperature to analyze experimental data of polystyrene in cyclohexane $[9,23]$. The theory of $A_{2}$, which was derived in the form of a scaling law, explained the observed molecular independence of $A_{2}$ and exhibited an increase through a minimum in the plot of $A_{2}$ vs temperature. The theoretical $A_{2}$ even became positive with further decrease of temperature. Both in the theory and in the plot in Fig. 3 the minimum appears near the region where the polymer dimension undergoes rapid change. This is also the case for polystyrene in cyclohexane [9]. The minimum in Fig. 3 could be compared with the theoretical one by using the values of $y=0.068$, $r=10^{-3} M_{w}=10^{3.38}$, and $\left\langle s^{2}\right\rangle=1.46 \times 10^{-11} \mathrm{~cm}^{2}$. The theoretical minimum due to the overlap approximation was about three times larger than the observed one.

\section{CONCLUSIONS}

Since a dilute solution of PMMA in the mixed solvent water + tert-butyl alcohol undergoes very slow phase separation, the mean square radius $\left\langle s^{2}\right\rangle$ and the second virial coefficient $A_{2}$ could be determined below the phaseseparation temperature by the usual light scattering measurements. The expansion factor obtained below the $\theta$ temperature was compared in an analytical way with a theory in which different segment distributions were used for $\alpha>1$ and $\alpha<1$. The coil-globule transition was revealed by the fact that $\alpha^{3} \tau M_{w}^{1 / 2}$ approached a constant with decreasing temperature. The transition point from coil to globule could not be determined uniquely because of the continuous change of $\alpha$ but the crossover points was suggested. In the plot of $A_{2}$ vs temperature a minimum occurred in the temperature region where the globule state began to be predominant. The theoretical calculation made by Tanaka also predicts a minimum in $A_{2}$. The theory and the present data were different in the behavior below the temperature where the minimum appeared. Since the mixed solvent water+tert-butyl alcohol did not show preferential sorption for the PMMA coil, the coil-globule transition of PMMA is also expected in a single solvent. The very slow phase separation observed for the present system may be caused primarily by the characteristic properties of the PMMA coil, because dilute solutions of PMMA in 1-chlorobutane also show slow phase separation [24]. The rate of the phase separation, which was most rapid near $34^{\circ} \mathrm{C}$, should be studied in connection with the behavior of $A_{2}$ and $\left\langle s^{2}\right\rangle$.
[1] P. J. Flory, Principles of Polymer Chemistry (Cornell University Press, Ithaca, 1953).

[2] O. B. Ptitsyn, A. K. Kron, and Y. Y. Eizner, J. Polym. Sci. Part C 16, 3509 (1968).
[3] P. G. DeGennes, J. Phys (Paris) Lett. 36, L55 (1975).

[4] I. M. Lifshitz, A. Yu. Grosberg, and A. R. Khokhlov, Rev. Mod. Phys. 50, 683 (1978).

[5] C. B. Post and B. H. Zimm, Biopolymers 18, 1487 (1979). 
[6] I. C. Sanchez, Macromolecules 12, 980 (1979).

[7] T. M. Birshtein and V. A. Pryamitsyn, Macromolecules 24, 1554 (1991).

[8] A. Yu. Grosberg and D. V. Kuznetsov, Macromolecules 25, 1970 (1992); 25, 1980 (1992); 25, 1991 (1992).

[9] M. Nierlich, J. P. Cotton, and B. Farnoux, J. Chem. Phys. 69, 1379 (1978).

[10] I. Nishio, S. T. Sun, G. Swislow, and T. Tanaka, Nature 281, 208 (1979).

[11] G. Swislow, S. T. Sun, I. Nishio, and T. Tanaka, Phys. Rev. Lett. 44, 796 (1980).

[12] S. T. Sun, I. Nishio, G. Swislow, and T. Tanaka, J. Chem. Phys. 73, 5971 (1980).

[13] P. Stepanek, C. Konak, and B. Sedlacek, Macromolecules 15, 1214 (1982).

[14] K. Kubota, S. Fujishige, and I. Ando, J. Phys. Chem. 94, 5154 (1990).

[15] I. H. Park, Q. W. Wang, and B. Chu, Macromolecules 20,
1965 (1987).

[16] B. Chu, I. H. Park, Q. W. Wang, and C. Wu, Macromolecules 20, 2833 (1987).

[17] J. Yu, Z. Wang, and B. Chu, Macromolecules 25, 1618 (1992).

[18] A. Yu. Grosberg and D. V. Kuznetsov, Macromolecules 25, 1996 (1992).

[19] F. Tanaka, J. Chem. Phys. 82, 4707 (1985).

[20] M. Nakata and N. Numasawa, Macromolecules 18, 1736 (1985).

[21] J. M. G. Cowie, M. A. Mohsin, and I. J. McEwen, Polymer 28, 1569 (1987).

[22] M. Nakata, K. Kawate, and Y. Ishitaka, Macromolecules 27, 1825 (1994).

[23] Z. Tong, S. Ohashi, Y. Einaga, and H. Fujita, Polymer J. 15, 835 (1983).

[24] M. Nakata and K. Kawate, Phys. Rev. Lett. 68, 2176 (1992). 